\title{
Article \\ Different Object Functions for SWIPT Optimization by SADDE and APSO
}

\author{
Wei Chien ${ }^{1}\left(\mathbb{0}\right.$, Chien-Ching Chiu ${ }^{2, *} \mathbb{C}$, Po-Hsiang Chen ${ }^{2} \oplus$, Yu-Ting Cheng ${ }^{2}$, Eng Hock Lim ${ }^{3}$, Yue-Li Liang ${ }^{1}$ \\ and Jia-Rui Wang ${ }^{4}$
}

check for updates

Citation: Chien, W.; Chiu, C.-C.; Chen, P.-H.; Cheng, Y.-T.; Lim, E.H.; Liang, Y.-L.; Wang, J.-R. Different Object Functions for SWIPT Optimization by SADDE and APSO. Symmetry 2021, 13, 1340. https:/ / doi.org/10.3390/sym13081340

Academic Editor: Aviv Gibali

Received: 4 July 2021

Accepted: 23 July 2021

Published: 24 July 2021

Publisher's Note: MDPI stays neutral with regard to jurisdictional claims in published maps and institutional affiliations.

Copyright: (c) 2021 by the authors. Licensee MDPI, Basel, Switzerland. This article is an open access article distributed under the terms and conditions of the Creative Commons Attribution (CC BY) license (https:// creativecommons.org/licenses/by/ $4.0 /)$.
1 Department of School of Electric and Information Engineering, Beibu Gulf University, Qinzhou 535000, China; air180@seed.net.tw (W.C.); rc@bbgu.edu.cn (Y.-L.L.)

2 Department of Electrical and Computer and Engineering, Tamkang University, New Taipei City 251301, Taiwan; 608440235@gms.tku.edu.tw (P.-H.C.); 802440031@gms.tku.edu.tw (Y.-T.C.)

3 Department of Electrical and Electronic, University Tunku Abdul Rahman, Selangor 43000, Malaysia; limeh@utar.edu.my

4 Department of Oceanography and Space Informatics, China University of Petroleum, Qingdao 266580, China; hankaixu@bbgu.edu.cn

* Correspondence: chiu@ee.tku.edu.tw

\begin{abstract}
Multiple objective function with beamforming techniques by algorithms have been studied for the Simultaneous Wireless Information and Power Transfer (SWIPT) technology at millimeter wave. Using the feed length to adjust the phase for different objects of SWIPT with Bit Error Rate (BER) and Harvesting Power (HP) are investigated in the broadband communication. Symmetrical antenna array is useful for omni bearing beamforming adjustment with multiple receivers. SelfAdaptive Dynamic Differential Evolution (SADDE) and Asynchronous Particle Swarm Optimization (APSO) are used to optimize the feed length of the antenna array. Two different object functions are proposed in the paper. The first one is the weighting factor multiplying the constraint BER and HP plus HP. The second one is the constraint BER multiplying HP. Simulations show that the first object function is capable of optimizing the total harvesting power under the BER constraint and APSO can quickly converges quicker than SADDE. However, the weighting for the final object function requires a pretest in advance, whereas the second object function does not need to set the weighting case by case and the searching is more efficient than the first one. From the numerical results, the proposed criterion can achieve the SWIPT requirement. Thus, we can use the novel proposed criterion (the second criterion) to optimize the SWIPT problem without testing the weighting case by case.
\end{abstract}

Keywords: simultaneous wireless information and power transfer (SWIPT); bit error rate (BER); evolution algorithms; harvesting power $(\mathrm{HP})$; ray-tracing

\section{Introduction}

The exponential growth of wireless devices has been developed quickly with the Internet of Things (IoT) in recent years. The fifth generation (5G) cellular networks have used the green field at millimeter wave to solve the problem of scarce spectrum resources due to the massive device access [1-6]. Other than the frequency resources, 5G can improve both the information and the energy efficiencies for the IoT. Since IoT devices are deployed at fixed locations and low-power environments, the wireless harvesting power has been investigated for IoT recently. To achieve this goal, a combination of millimeter wave ( $\mathrm{mm}$ Wave) communication and energy harvesting for SWIPT had been studied in [7-20]. In [21], multiple objective functions were considered to optimize the communication quality, but the authors need to find the best weighting in advance for those cases. In [9], the authors had derived analytical expressions to optimize the power harvesting performance at mmWave communication with low-power devices. In [22], the optimization of energy efficiency for a SWIPT, non-orthogonal multiple access was investigated under the stochastic. In [23], 
the optimal analytic solution with the Signal to Interference plus Noise Ratio (SINR) constraint was presented for the narrow band signal.

To the best of our knowledge, most papers utilize a narrow band system under the SINR constraint with an optimal analytic solution. For wide band signals, the BER and SINR are not completely correlated. The Bit Error Rate (BER) performance shall be considered in the wireless wideband communication system due to the frequency selective effect of the multipath channel and Inter Symbol Interference (ISI) rather than SINR only. In our past research, we attempt to solve this problem with a multiple objective function. In [21], we use the single objective function value to set the weighting for the multiple function. In other words, we cannot find the suitable multiple function unless the maximum single objective function has been found. In [24], we try to use the constraints for optimization. However, the penalty for the algorithm constraint should be adjusted according to the environment. Therefore, we try to improve the objective function for easy adjustment. In this paper, we introduce different objective functions for the BER and power harvesting efficiency for a wideband circular array SWIPT system. We use a novel multiple objective function that does not need to set specific weighting for the BER constraint objective function.

The system model and the deployment of the antenna array are presented in Section 2. Section 3 explains the SADDE and APSO with the objective function. In Section 4, two different multiple objective functions with APSO and SADDE are compared. The optimization of the object function is achieved. The configured parameters and the SWIPT quality are compared by numerical simulation. The conclusion section summarizes the key points of this paper.

\section{System Model}

\subsection{Channel Model}

For any environment, we compute the frequency response by the ray-tracing method at millimeter wave communication $[25,26]$. The ray-tracing program sums the total ray images as follows

$$
H(f)=\sum_{i=1}^{N} \alpha_{i}(f) e^{j \psi_{i}(f)}
$$

where $H$ is the frequency response for the summation of the total path $N . f$ is the frequency and $i$ is the path index of the ray. $\alpha_{i}$ is the $i$-th receiving magnitude consisting the phase information and the intensity. $\psi_{i}(f)$ is the phase shift according to the time delay. The time domain impulse of the equivalent baseband can be transformed by the inverse fast Fourier transform and it can be composed as follows,

$$
h(t)=\int_{-\infty}^{\infty} H(f) e^{j 2 \pi f t} d f
$$

where $h(t)$ is the impulse response.

\subsection{SWIPT}

Figure 1 shows that the power-splitter block is used for the SWIPT system. The received signals are divided into two streams for the RF energy harvester and the information receiver with various power levels.

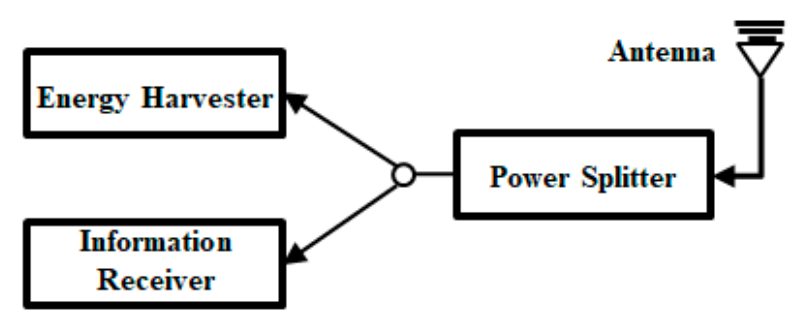

Figure 1. The splitter architecture for the SWIPT system. 
The harvested wireless powered is modeled as

$$
H P=\eta \int_{-\infty}^{\infty} h(t)^{2} d t
$$

where $\eta$ is the portion of RF signals utilized for power gathering. The information decoder quality BER is used to evaluate the inter-symbol-interference for the SWIPT system and can be computed as the following formula [26]:

$$
B E R=\sum_{s=1}^{2^{s}} P\left(\vec{d}_{s}\right) \cdot \frac{1}{2} \operatorname{erf} c\left[\frac{V\left(t=s T_{d}\right)}{\sqrt{2} \sigma} \cdot\left(d_{s}\right)\right]
$$

where $\operatorname{erf} c(x)=\frac{2}{\sqrt{\pi}} \int_{x}^{\infty} e^{-y^{2}} d y$ is the complementary error function and $\left\{\vec{d}_{s}\right\}=\left\{d_{1}, d_{2}, \ldots d_{S}\right\}$ is the binary sequence.

$$
S N R_{R}=S N R_{T} \times V
$$

\subsection{Antenna Array}

The deployment of the transmitting antenna array is shown in Figure 2 and we apply a beamforming technique to focus the intensity to reduce the path loss in the environment. The circular antenna array is useful for adjustment with multiple receivers, as the deployment is symmetrical.

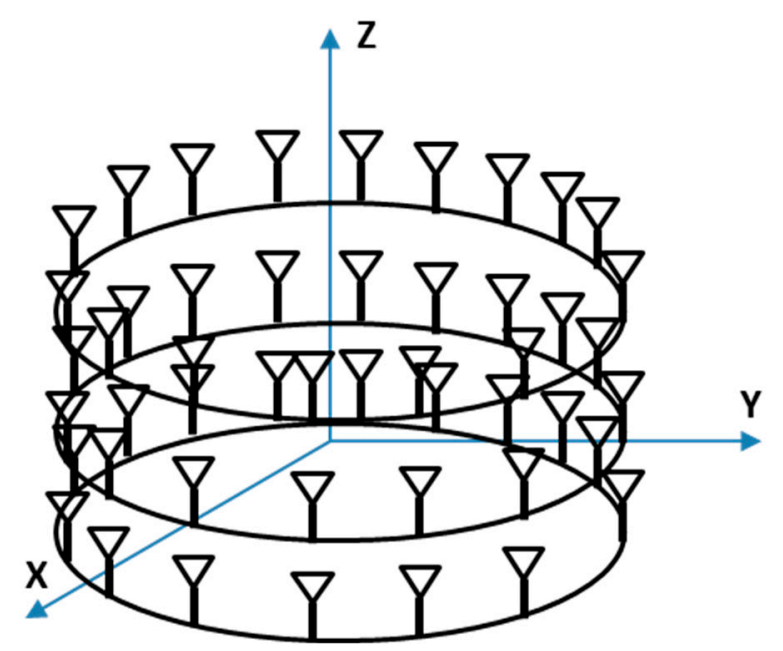

Figure 2. The deployment of antenna array.

The array factor is used to calculate the field and can be derived as [27]

$$
A F(\theta, \phi, f)=\sum_{n=1}^{M} W_{n} e^{j k r\left[\sin \theta \sin \theta_{n} \cos \left(\phi-\phi_{n}\right)+\cos \theta_{n} \cos \theta\right]}
$$

where $\theta$ and $\phi$ are the spherical coordinates system. $\theta_{n}, \phi_{n}$ and $r$ are the elevation angle, azimuth angle and the distance of the antenna position respectively. $M$ is the total number of antennas in the antenna array. $k$ is the wavenumber. The adjustment of phase delay and magnitude power can be expressed as

$$
W_{m}=I_{m} e^{j D_{m}(f)}
$$

where $I_{m}$ and $D_{m}(f)$ are the excitation current and the phase delay. The relation of feed length and $D_{m}(f)$ can be expressed as

$$
D_{m}(f)=-\frac{2 \pi f}{c} \sqrt{\varepsilon_{r}} \cdot \ell_{n}
$$


where $\varepsilon_{r}$ is the relative permittivity of feed line and $\ell_{n}$ is the feed length used to adjust the phase delay. $c$ is the light speed.

\section{Evolution Algorithm}

In this paper, we use two different evolution algorithms to compare the multiple objective functions. The first evolution algorithm is APSO $[21,27,28]$, which is developed from the Particle Swarm Optimization (PSO). PSO was inspired by the social behavior of animals as fish schooling. APSO adjusts the velocity when the best object function is updated, and it converges more rapidly than PSO. As shown in Figure 3, there are six main steps for APSO optimization.

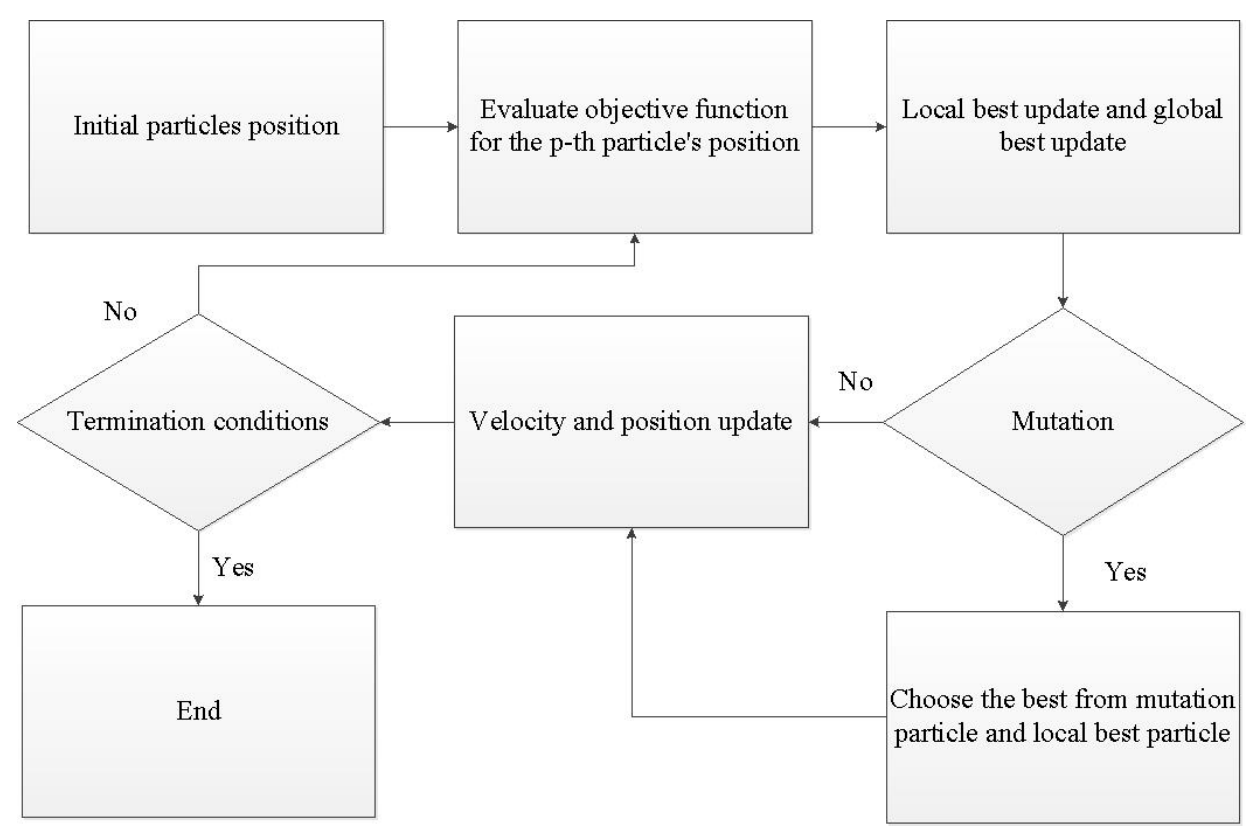

Figure 3. APSO flow chart.

Step 1. We first set all particles with fixed range which depends on the feed length of the antenna array. The algorithm initializes particle position randomly with d dimensional adjustment.

Step 2. Evaluate the $p$-th object value of the objective function, where $p$ is the number sequence in the total population.

Step 3. Update global best particle and local best particle.

Step 4. The particles are mutated with ten percent probability. Then update the local best particle and global best particle according to the objective value.

Step 5. Velocity and position are updated according to the position of the local best and global best particle. Step 6. To step 2 or stop operating the algorithm when the present generation is reached.

The SADDE is developed from Dynamic Differential Evolution (DDE) [21,26,29]. In this research, we add some mechanisms to let the adjustment factor self-adapt during the searching. The flowchart of the SADDE algorithm is shown in Figure 4.

Step 1. We load the setting parameter and initiate the parameter of population randomly. Every population has d dimensional adjustment parameters for the antenna array and those parameters are employed to compute the objective function.

Step 2. Calculate the objective function and update the best value of the local particle based on the p-th particle's adjustment parameters.

Step 3. Mutate the trial vector according to the control vector and adjust the control vector for the next generation.

Step 4. By the preset probability, decide whether to start the crossover mechanism.

Step 5. Update the position of the global best particle. 
Step 6. According to the total number of particles and the number of generations, go to Step 2. or stop operating the algorithm.

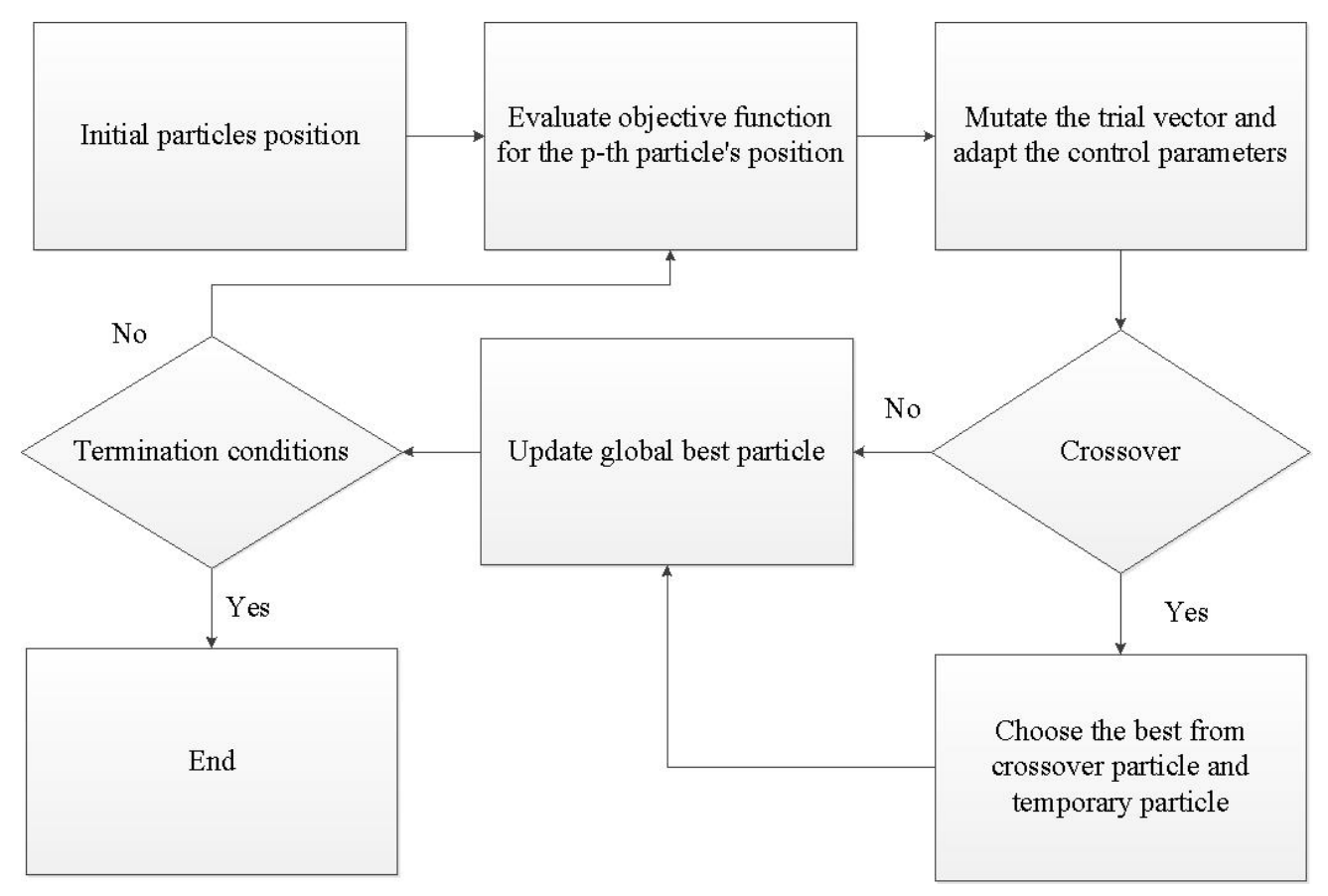

Figure 4. SADDE flow chart.

Here, consider two different requirements at the same time. One is the $B E R$ and the other is the total harvesting power. If $B E R$ is constrained at $10^{-5}$, we define $B E R_{w}$ as Equation (8) in order to optimize the objection functions into one.

$$
B E R_{W}=\left\{\begin{array}{cl}
\frac{10^{-5}}{B E R} & , B E R>10^{-5} \\
1 & , B E R<10^{-5}
\end{array}\right.
$$

The total power harvesting can be expressed as

$$
H P_{T}=\sum_{i=1}^{N_{R}} H P_{i}
$$

where $N_{R}$ is the total number of receivers in the environment. $H P_{T}$ is expected to be as large as possible. The above two functions have been combined with two different methods. In the first proposed criterion, we set $B E R_{W}$ multiplied by $H P_{T}$ plus $H P_{T}$ as the objective function. Thus, $B E R_{W}$ multiplied by $H P_{T}$ and $H P_{T}$ has the similar order if the BER is less than $10^{-5}$. However, from the numerical simulations, this criterion cannot reach the BER constraint of $10^{-5}$. As a result, we introduce the weighting factor $W$ in the objective function, as show in Equation (9)

$$
1 /\left(W \times B E R_{W} \times H P_{T}+H P_{T}\right)
$$

However, you must test the weighting factor $\mathrm{W}$ case by case beforehand in order to obtain satisfied results. Hence, we propose a second criterion in Equation (10) that only keeps the terms $B E R_{W}$ multiplied by $H P_{T}$ where $\mathrm{W}$ for balancing the required value can be skipped.

$$
1 /\left(B E R_{W} \times H P_{T}\right)
$$




\section{Numerical Results}

In this paper, the wide band system with frequencies ranging from $39 \mathrm{GHz}$ to $40 \mathrm{GHz}$ at millimeter wave are presented and the ray-tracing technique is used to calculate the corresponding channel. Figure 5 shows a simple office environment for simulation. There are three receivers $(\mathrm{R} x 1, \mathrm{R} \times 2, \mathrm{R} \times 3)$ and one transmitter $(\mathrm{Tx})$ in the office. The coordinates for $\mathrm{R} x 1, \mathrm{R} \times 2$ and $\mathrm{R} \times 3$ are $(3.8,8.8,1.0) \mathrm{m},(3.0,7.5,1.0) \mathrm{m}$ and $(3.0,5.5,1.0) \mathrm{m}$ respectively. The transmitter is at $(7.0,6.0,1.0) \mathrm{m}$ in the office. We consider three layers of circular arrays with a vertical distance of $1 / 4$ wavelength. Each layer has 16 wide band short dipole antennas. In other words, there are 48 short dipole antennas. The power harvesting nodes are $\mathrm{R} \times 2$ and $\mathrm{R} \times 3$. The SWIPT node is Rx1. The SWIPT system is used at Rx1 with the power-dividing architecture $(\eta=0.5)$. The transmission power to the noise ratio $\left(S N R_{T}\right)$ is set to be $33 \mathrm{~dB}$. We set the same population size and the same generation number for APSO and SADDE. The population is 60 and the iterations are 500. The mutation of APSO and crossover of SADDE are both with the same probability. The weighting $W$ for the objective function is set to 10 in Equation (9).

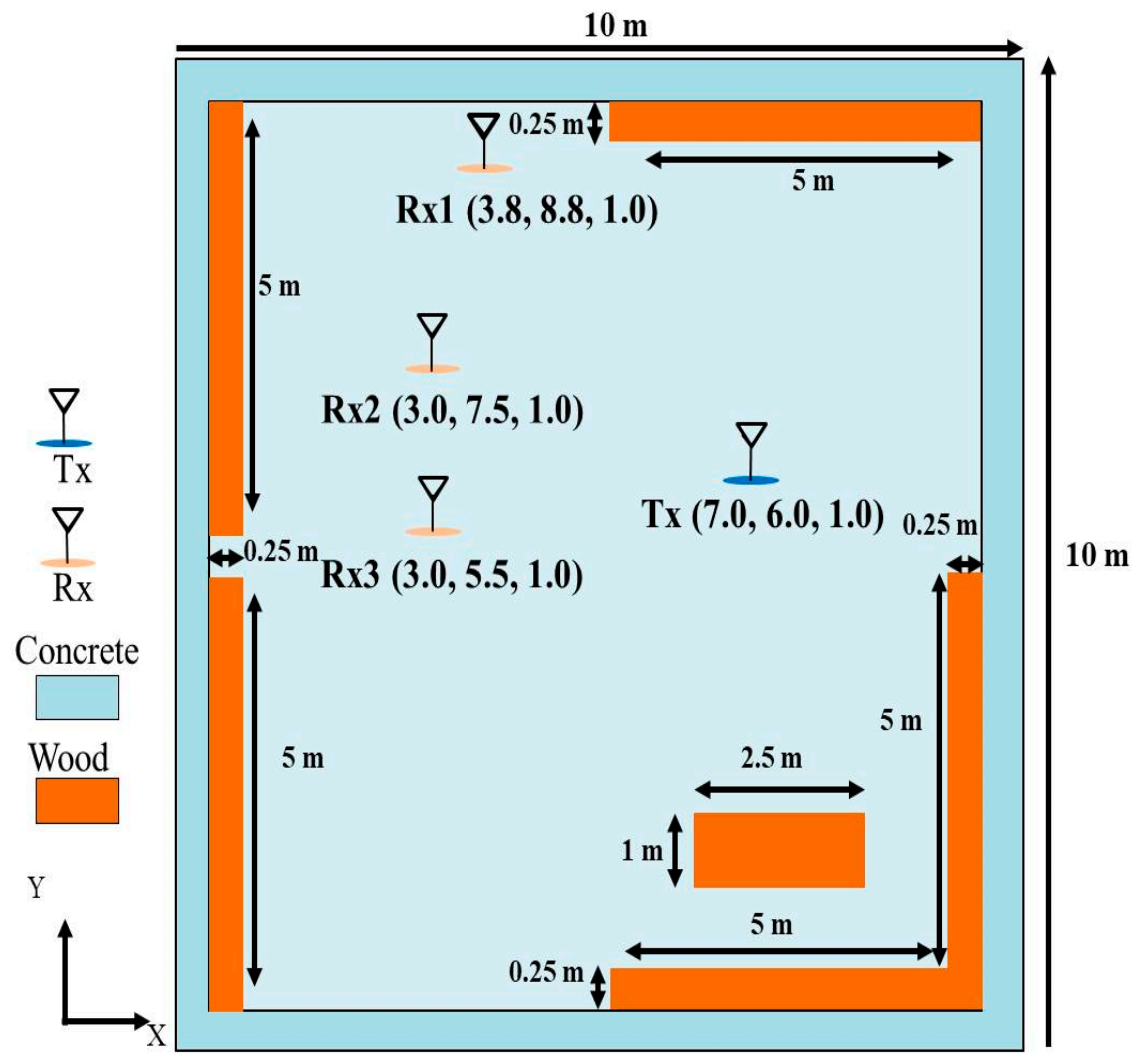

Figure 5. Indoor Environment Layout.

Figure 6 shows the effect of different objective functions for the BER at Rx1. From this figure, we can see that the first proposed criterion can achieve meet the BER constrain for both APSO and SADDE, but the convergent speed for APSO is better than SADDE's. This figure also tells us that the second proposed criterion can also achieve the goal of a large $\mathrm{HP}$ and meet the BER constraint as well. In addition to that, the convergent speeds are similar for both APSO and SADDE. Figure 7 shows the total harvesting power ratio for different objective functions. Note that the harvesting power ratio is the power ratio with and without adjusting the feed length at the transmitter. From our simulation, the total harvesting power can be improved about 60 times from the initial array by applying on proposals. Furthermore, it is clear that the total harvesting power for the second proposed criterion is better than that for the first one proposed. Moreover, it is found that APSO converges faster than SADDE in the first proposed criterion. 


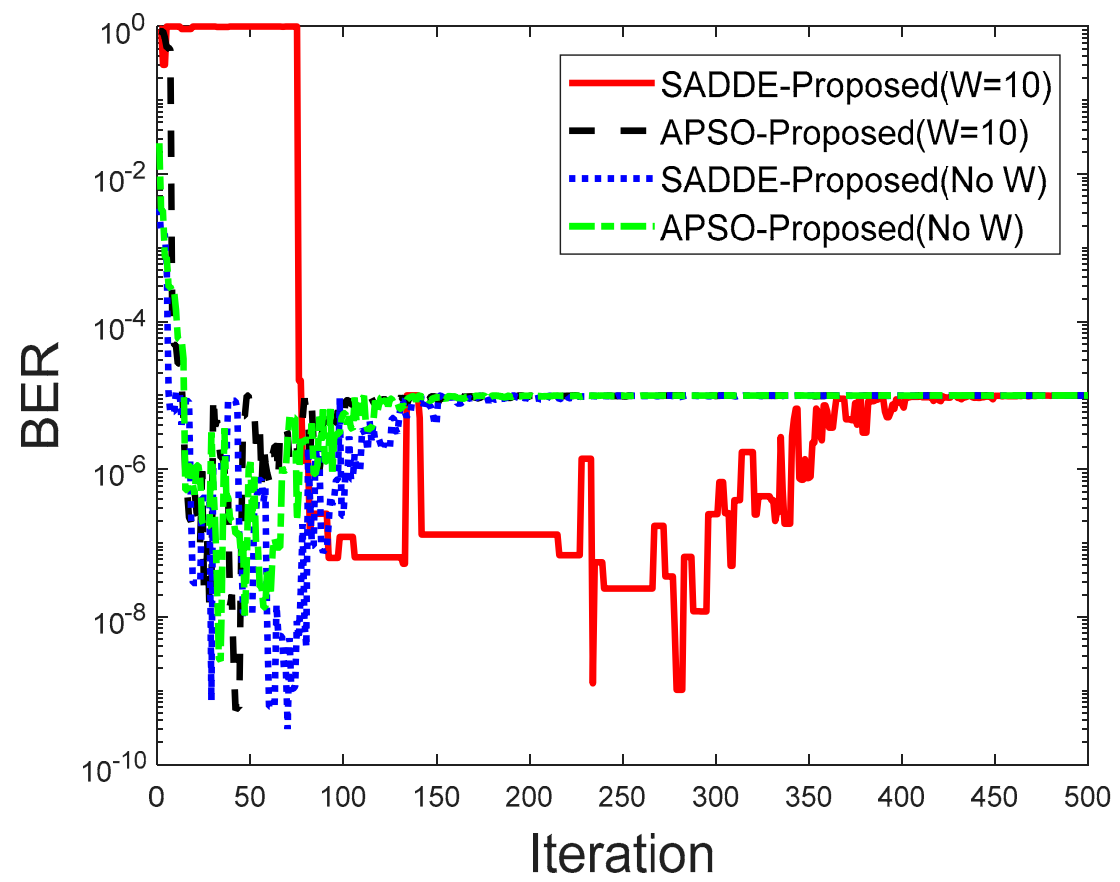

Figure 6. BER for different objective functions.

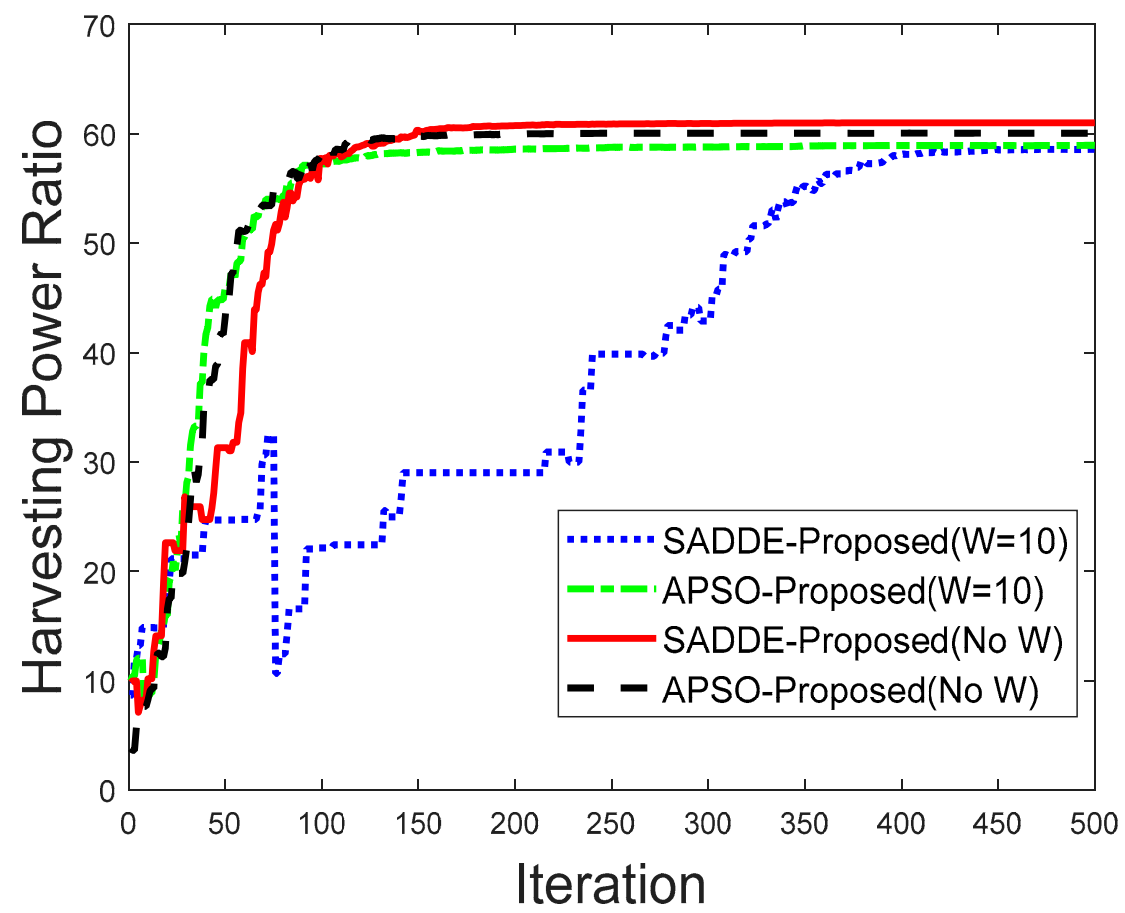

Figure 7. Harvesting power ratios for different objective functions.

Figures 8 and 9 show the radiation patterns of the first proposed criterion by two different algorithms. Compare Figures 8 and 9, and refer to Figure 5 that indicates the location of the receivers and the transmitter. The adjustment of beams has all been directed to Rx1, Rx2 and Rx3 by SADDE and APSO. However, it is seen that the pattern for Rx3 is the largest compared to the other two receivers. This is because $\mathrm{Rx} 3$ is closest to the transmitter. While increasing the total harvesting power, the antenna gain for Rx3 is the largest. On the contrary, the pattern for Rx1 is the smallest. Due to the power being split at Rx1, the algorithm is adjusted to use minimum power to meet the BER criterion. As a result, the gain for Rx1 is smallest compared to Rx2 and Rx3. Note that both SNR and multi-path with 
ISI will affect the BER at the same time. Figures 10 and 11 show the radiation patterns for the $\mathrm{w} /$ proposed criterion by two different algorithms. It also achieves the BER constraint and improves the total harvesting power. From all the above results, we can conclude that the second proposed criterion is more efficient for the SWIPT system and setting the weighting factor is not necessary.

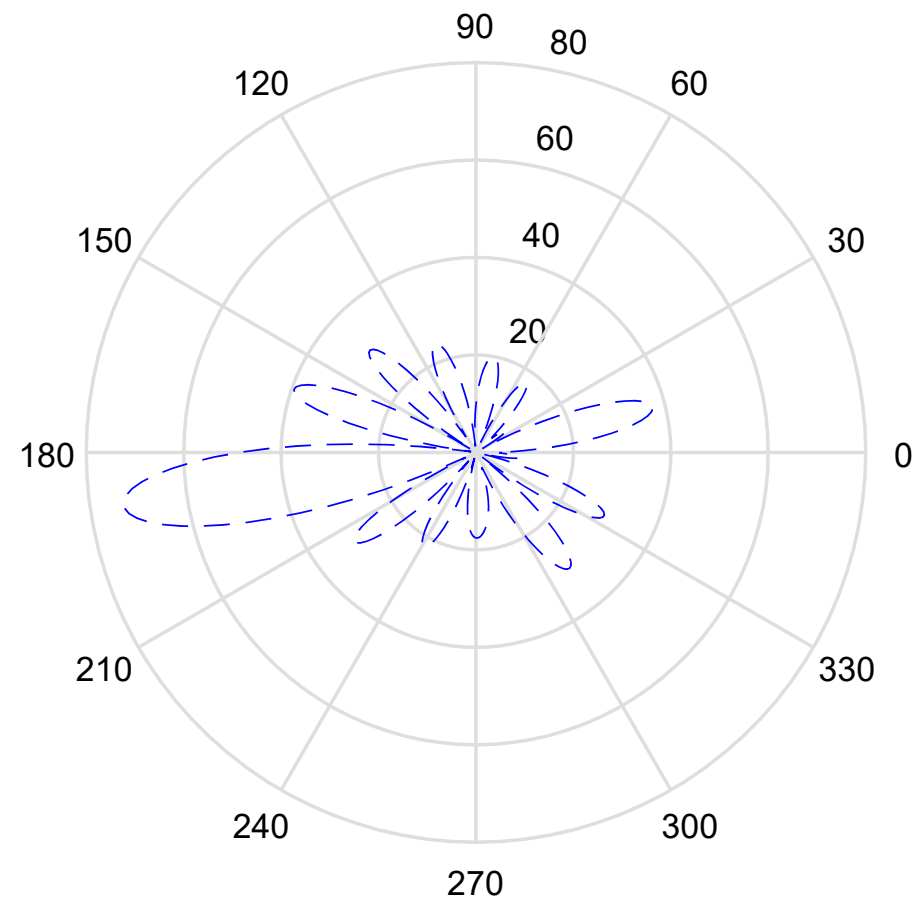

Figure 8. Antenna pattern by SADDE for the first proposed criterion.

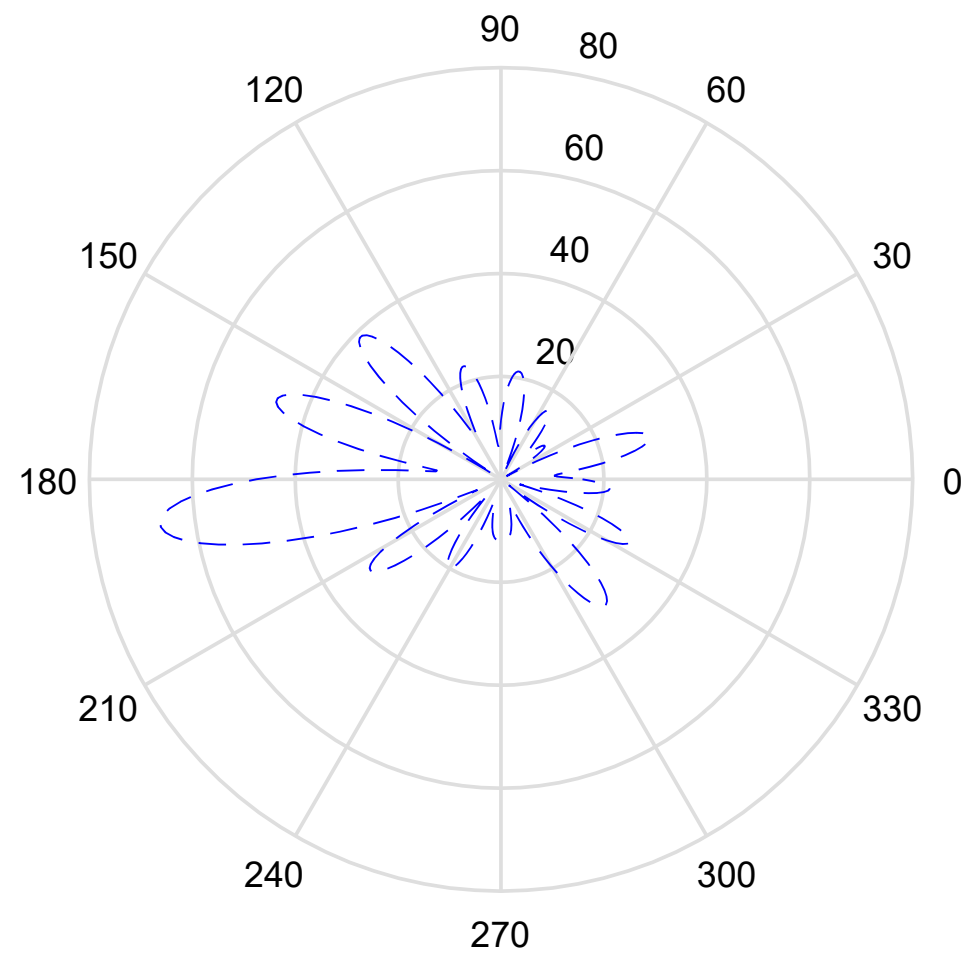

Figure 9. Antenna pattern by APSO for the first proposed criterion. 


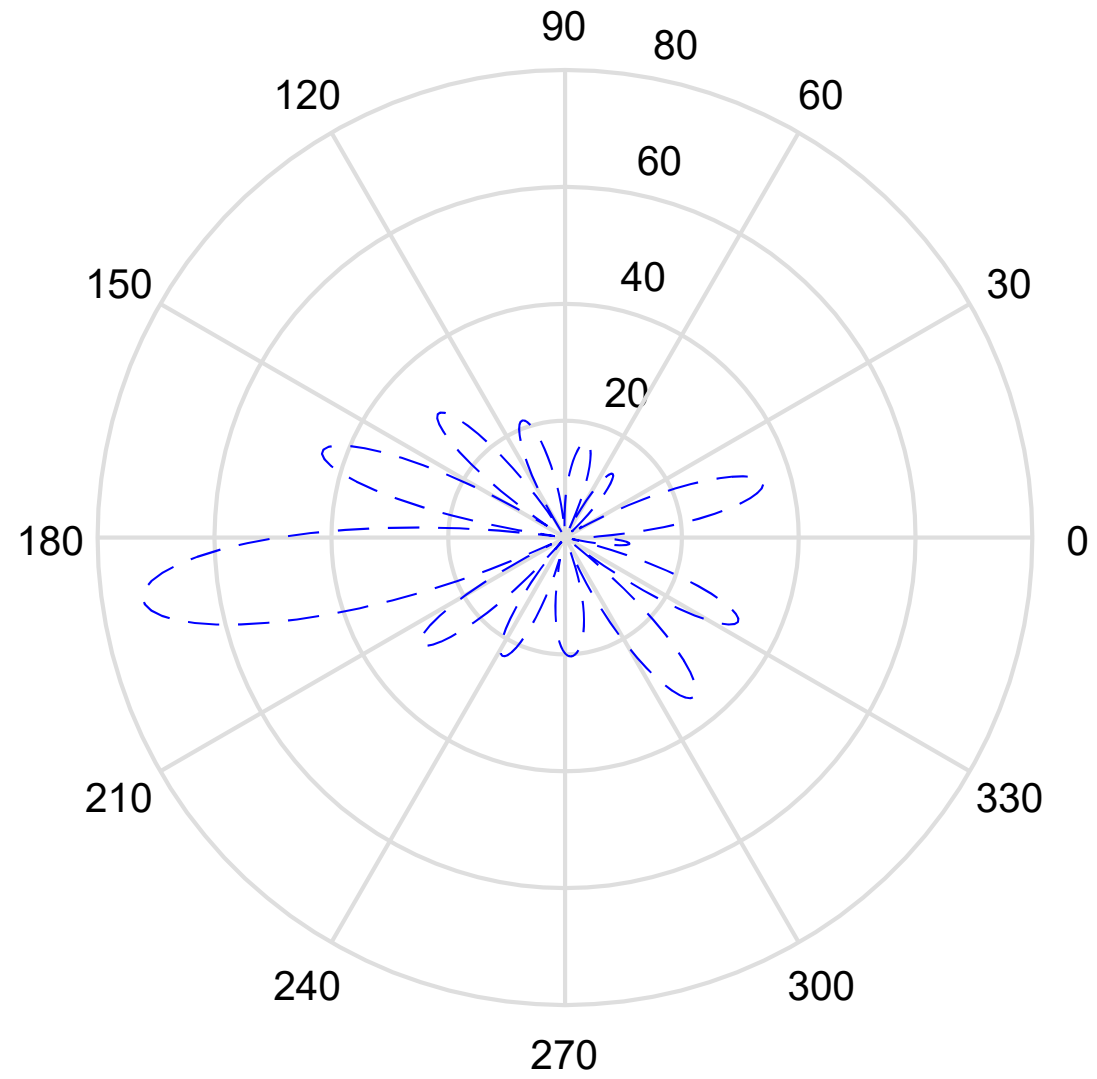

Figure 10. Antenna pattern by SADDE for the second proposed criterion.

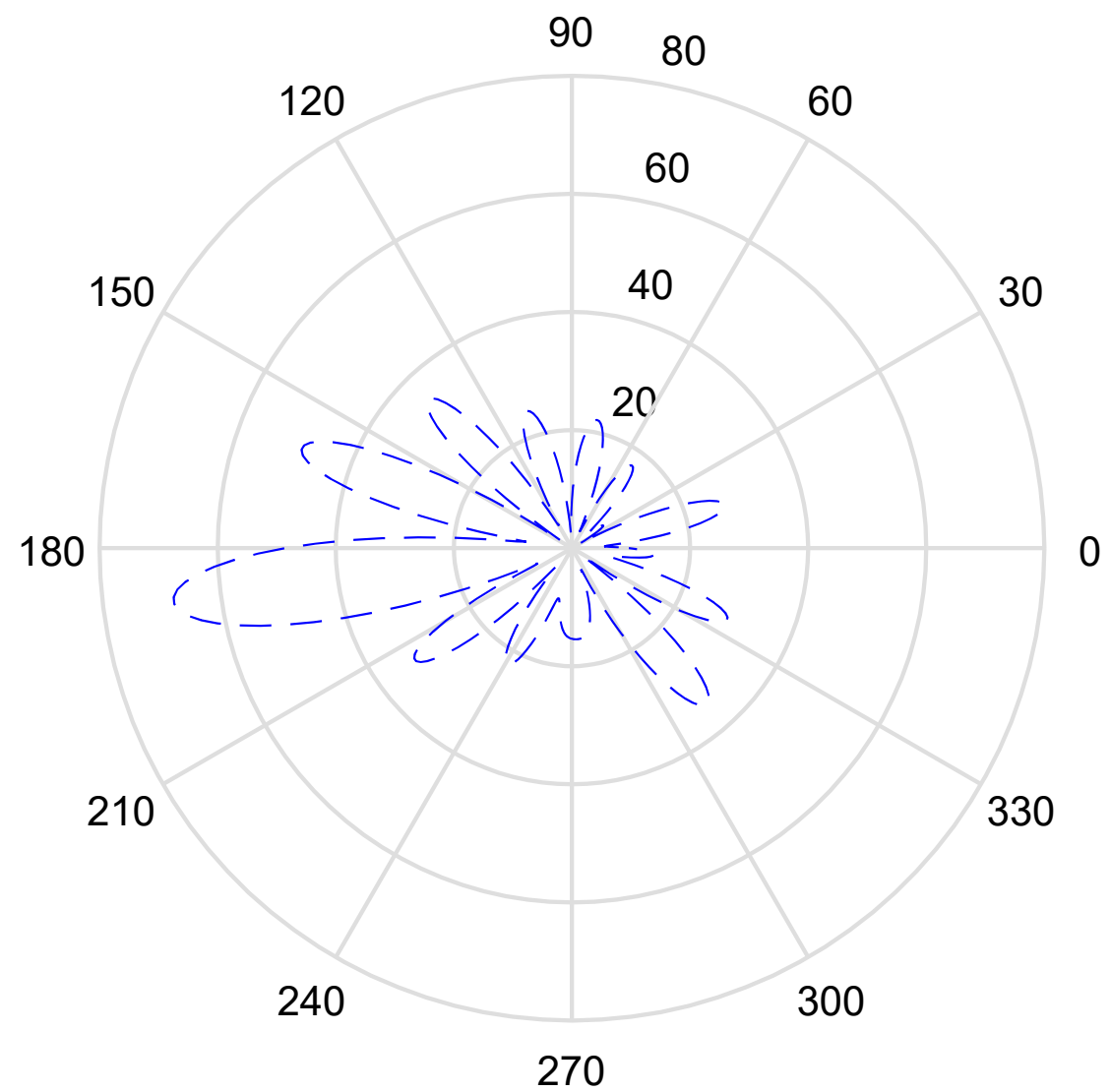

Figure 11. Indoor Environment Layout. 
In the above results, the first objective function is chosen to let two objective values with the same order of magnitude and we also give a weighting $W$ for adjustment $\left(1 /\left(W \times B E R_{W} \times H P_{T}+H P_{T}\right)\right)$. However, the weighting must be chosen in advance, with a suitable value for the best optimization. Thus, we attempt to remove $W$ and the plus term $H P_{T}$ to keep only the $B E R_{W} \times H P_{T}$ term. Numerical results show that similar optimization results can be found for these two objective functions. However, the second objective function does not need to find the best $W$ in advance for optimization. Hence, the second function is better for the optimization problem in this paper.

\section{Conclusions}

In this paper, different algorithms and different criteria are used to optimize the harvesting power and information quality for the SWIPT system at millimeter wave in the real environment. SADDE and APSO are used to optimize the SWIPT system and compare different types of objective functions. For the SWIPT system, we consider BER and HP for information quality and harvesting power. The first criterion (Equation (9)) can achieve the BER constraint and in the meantime optimize the total harvesting power. APSO has a better convergent speed than SADDE but has the same harvesting power. Although the first criterion can achieve the goal for SWIPT, the criterion must test the weighting in advance to obtain good results. However, the second proposed criterion does not need to set the weighting case by case and this leads to more efficient searching. From the numerical results, we can see that the second proposed criterion is capable of finding the optimized harvesting power under the BER constraint. Thus, we can use the novel proposed criterion (the second criterion) to optimize the SWIPT problem without having to test the weighting case by case in advance. For all these, we finally conclude that our proposed criterion is novel and effective for the SWIPT system.

Author Contributions: Conceptualization, W.C. and C.-C.C.; methodology, W.C. and P.-H.C.; software, C.-C.C. and Y.-T.C.; validation, E.H.L., Y.-L.L. and J.-R.W.; formal analysis, C.-C.C. and P.-H.C.; investigation, Y.-T.C. and E.H.L.; resources, W.C. and J.-R.W.; data curation, Y.-L.L. and W.C.; writingoriginal draft preparation, Y.-T.C. and P.-H.C.; writing-review and editing, C.-C.C.; visualization, E.H.L.; supervision, W.C.; project administration, C.-C.C.; funding acquisition, W.C. All authors have read and agreed to the published version of the manuscript.

Funding: This research was funded by Guangxi Higher Vocational Education Reform Project (GXGZJG2020A001) and Undergraduate Innovation and Entrepreneurship Project (2021).

Institutional Review Board Statement: Not applicable.

Informed Consent Statement: Not applicable.

Conflicts of Interest: The authors declare no conflict of interest.

\section{References}

1. Ansari, R.I.; Chrysostomou, C.; Hassan, S.A.; Guizani, M.; Mumtaz, S.; Rodriguez, J.; Rodrigues, J.J.P.C. 5G D2D Networks: Techniques, Challenges, and Future Prospects. IEEE Syst. J. 2018, 12, 3970-3984. [CrossRef]

2. Hossain, E.; Rasti, M.; Tabassum, H.; Abdelnasser, A. Evolution toward 5G multi-tier cellular wireless networks: An in-terference management perspective. IEEE Wirel. Commun. 2014, 21, 118-127. [CrossRef]

3. Shafique, K.; Khawaja, B.A.; Sabir, F.; Qazi, S.; Mustaqim, M. Internet of Things (IoT) for Next-Generation Smart Systems: A Review of Current Challenges, Future Trends and Prospects for Emerging 5G-IoT Scenarios. IEEE Access 2020, 8, 23022-23040. [CrossRef]

4. Zhou, B.; Liu, A.; Lau, V.K.N. Successive Localization and Beamforming in 5G mmWave MIMO Communication Systems. IEEE Trans. Signal Process. 2019, 67, 1620-1635. [CrossRef]

5. Zhao, X.; Du, F.; Geng, S.; Sun, N.; Zhang, Y.; Fu, Z.; Wang, G. Neural network and GBSM based time-varying and stochastic channel modeling for $5 \mathrm{G}$ millimeter wave communications. China Commun. 2019, 16, 80-90. [CrossRef]

6. Park, J.J; Moon, J.H.; Lee, K.Y.; Kim, D.I. Transmitter-Oriented Dual-Mode SWIPT With Deep-Learning-Based Adaptive Mode Switching for IoT Sensor Networks. IEEE Internet Things J. 2020, 7, 8979-8992. [CrossRef]

7. Varshney, L.R. Transporting information and energy simultaneously. In Proceedings of the 2008 IEEE International Symposium on Information Theory, Toronto, ON, Canada, 6-11 July 2008; pp. 1612-1616. 
8. Wang, X.; Gursoy, M.C. Coverage Analysis for Energy-Harvesting UAV-Assisted mmWave Cellular Networks. IEEE J. Sel. Areas Commun. 2019, 37, 2832-2850. [CrossRef]

9. Khan, T.A.; Alkhateeb, A.; Heath, R.W. Millimeter Wave Energy Harvesting. IEEE Trans. Wirel. Commun. 2016, 15, 6048-6062. [CrossRef]

10. Zhang, J.; Zheng, G.; Krikidis, I.; Zhang, R. Fast Specific Absorption Rate Aware Beamforming for Downlink SWIPT via Deep Learning. IEEE Trans. Veh. Technol. 2020, 69, 16178-16182. [CrossRef]

11. Lee, K.; Lee, W. Learning-Based Resource Management for SWIPT. IEEE Syst. J. 2020, 14, 4750-4753. [CrossRef]

12. Zhang, Z.; Lu, Y.; Huang, Y.; Zhang, P. Neural Network-Based Relay Selection in Two-Way SWIPT-Enabled Cognitive Radio Networks. IEEE Trans. Veh. Technol. 2020, 69, 6264-6274. [CrossRef]

13. Hu, Z.; Xie, D.; Jin, M.; Zhou, L.; Li, J. Relay Cooperative Beamforming Algorithm Based on Probabilistic Constraint in SWIPT Secrecy Networks. IEEE Access 2020, 8, 173999-174008. [CrossRef]

14. Li, Q.; Yang, L. Robust Optimization for Energy Efficiency in MIMO Two-Way Relay Networks With SWIPT. IEEE Syst. J. 2020, 14, 196-207. [CrossRef]

15. Liu, F.; Liu, Y.; Liu, Y.; Yu, J. Secure Beamforming in Full-Duplex Two-Way Relay Networks With SWIPT for Multimedia Transmission. IEEE Access 2020, 8, 26851-26862. [CrossRef]

16. Luo, J.; Tang, J.; So, D.K.C.; Chen, G.; Cumanan, K.; Chambers, J.A. A Deep Learning-Based Approach to Power Minimization in Multi-Carrier NOMA With SWIPT. IEEE Access 2019, 7, 17450-17460. [CrossRef]

17. Qi, Q.; Chen, X.; Ng, D.W.K. Robust Beamforming for NOMA-Based Cellular Massive IoT With SWIPT. IEEE Trans. Signal Process. 2020, 68, 211-224. [CrossRef]

18. Sun, X.; Yang, W.; Cai, Y.; Wang, M. Secure mmWave UAV-Enabled SWIPT Networks Based on Random Frequency Diverse Arrays. IEEE Internet Things J. 2021, 8, 528-540. [CrossRef]

19. Sun, X.; Yang, W.; Cai, Y.; Ma, R.; Tao, L. Physical Layer Security in Millimeter Wave SWIPT UAV-Based Relay Networks. IEEE Access 2019, 7, 35851-35862. [CrossRef]

20. Liu, R.; Li, H.; Guo, Y.; Li, M.; Liu, Q. Hybrid Beamformer Design with Low-Resolution Phase Shifters in MU-MISO SWIPT Systems. In Proceedings of the 2018 10th International Conference on Wireless Communications and Signal Processing (WCSP), Hangzhou, China, 18-20 October 2018; pp. 1-6.

21. Chiu, C.-C.; Tong, Y.-X.; Cheng, Y.-T. Comparison of self-adaptive dynamic differential evolution and particle swarm optimization for smart antennas in wireless communication. Int. J. Commun. Syst. 2019, 32, e3941. [CrossRef]

22. Tang, J.; Luo, J.; Liu, M.; So, D.K.C.; Alsusa, E.; Chen, G.; Wong, K.-K.; Chambers, J.A. Energy Efficiency Optimization for NOMA With SWIPT. IEEE J. Sel. Top. Signal Process. 2019, 13, 452-466. [CrossRef]

23. Hao, W.; Sun, G.; Chu, Z.; Xiao, P.; Zhu, Z.; Yang, S.; Tafazolli, R. Beamforming Design in SWIPT-Based Joint Multicast-Unicast mmWave Massive MIMO With Lens-Antenna Array. IEEE Wirel. Commun. Lett. 2019, 8, 1124-1128. [CrossRef]

24. Chien, W.; Hsieh, T.-T.; Chiu, C.-C.; Cheng, Y.-T.; Lee, Y.-H.; Chen, Q. Theoretical Derivation and Optimization Verification of BER for Indoor SWIPT Environments. Symmetry 2020, 12, 1185. [CrossRef]

25. Hsiao, A.-Y.; Yang, C.-F.; Wang, T.-S.; Lin, I.; Liao, W.-J. Ray tracing simulations for millimeter wave propagation in 5G wireless communications. In Proceedings of the 2017 IEEE International Symposium on Antennas and Propagation \& USNC/URSI National Radio Science Meeting, San Diego, CA, USA, 9-14 July 2017; pp. 1901-1902. [CrossRef]

26. Lai, G.D.; Chiu, C.C.; Chen, Y.T. BER reduction for ultra wideband multicasting system by beamforming techniques. J. Appl. Sci. Eng. 2018, 21, 587-594.

27. Chien, W.; Chiu, C.-C.; Cheng, Y.-T.; Lee, Y.-L. Evolution algorithms applied for 3D beamforming UWB channels. Microsyst. Technol. 2021, 27, 1797-1804. [CrossRef]

28. Chiu, C.-C.; Ho, M.-H.; Liao, S.-H. PSO and APSO for optimizing coverage in indoor UWB communication system. Int. J. RF Microw. Comput. Eng. 2012, 23, 300-308. [CrossRef]

29. Brest, J.; Greiner, S.; Bošković, B.; Mernik, M.; Zumer, V. Self-Adapting Control Parameters in Differential Evolution: A Comparative Study on Numerical Benchmark Problems. IEEE Trans. Evol. Comput. 2006, 10, 646-657. [CrossRef] 